\title{
Grande vitesse ferroviaire et développement local dans des contextes institutionnels différents : Pour une spatialisation des analyses de l'innovation de service
}

\author{
High-speed rail and local development in different institutional contexts:
} towards a spatialization of services innovation analyses

\section{Marie Delaplace ${ }^{1}$}

${ }^{1}$ Université de Paris-Est Marne-la-Vallée, Lab'urba, Ecole d'Urbanisme de Paris (EUP), marie.delaplace@u-pem.fr

RÉSUMÉ. La grande vitesse ferroviaire génère de nombreuses attentes en termes de développement local. Si tout le monde ou presque s'accorde pour souligner l'absence d'effets structurants des infrastructures de transport, il reste cependant à penser les déterminants de la relation entre grande vitesse ferroviaire et développement local. Cela est d'autant plus important que celle-ci se développe dans des pays émergents ou en développement, i.e. dans des contextes institutionnels et sociaux différents. A partir de la littérature relative à l'innovation dans les services, cet article propose de dépasser l'analyse des effets de l'infrastructure en elle-même, pour se centrer sur les services qu'elle rend possibles. II propose de considérer que la relation de services diffère dans le temps et dans l'espace. L'analyse en termes d'innovation de services doit alors être spatialisée. Les technologies, les caractéristiques de services, les ressources des clients et des prestataires, de même que les règles qui encadrent leurs actions sont en effet inscrites dans des sociétés données.

ABSTRACT. Expectations in cities served by high-speed rail in terms of local economic development are numerous. While nearly everyone would agree that the structuring effects of transportation infrastructures don't exist, the factors that influence the link between high-speed rail and local development need to be analyzed. This is all the more important because high-speed lines are currently built in emerging or developing countries, in other words in very different institutional and social contexts. Based on the literature which focuses on service innovations, this article intends to go beyond an analysis concerning the infrastructure to focus on the services that it enables. It highlights that the services relationship varies in time and in space. Consequently, the analysis of service innovation must be spatialized. Indeed, technologies, services characteristics, clients and provider resources, as well as the rules guiding their action are embedded in given societies.

MOTS-CLÉS. Grande vitesse ferroviaire, Développement économique local, Services, Innovation.

KEYWORDS. High-speed rail, Local economic development, Services, Innovation.

\section{Introduction}

Au 1er janvier 2016, on comptait $34679 \mathrm{~km}$ de lignes ferroviaires à grande vitesse dans le monde et $15790 \mathrm{~km}$ étaient en construction. Plus de $35000 \mathrm{~km}$ sont également projetés à l'horizon 2030 et ce, dans de nombreux pays. Ces dessertes génèrent de nombreuses attentes en termes de développement économique local. Cependant tout le monde ou presque s'accorde pour dépasser l'analyse en termes d'effets structurants des infrastructures de transport : il n'y a pas de relation de causalité entre infrastructure et développement local (cf. le débat dans les controverses de l'Espace géographique, 2014). La question est alors de comprendre les interactions entre grande vitesse ferroviaire et développement local. Cette question est d'autant plus importante que le XXIème siècle est caractérisé par l'extension du réseau dans des pays émergents ou en développement (Chine, Inde, Turquie, Brésil, Maroc, Malaisie, Egypte, etc.). La grande vitesse ferroviaire s'inscrit alors dans des contextes institutionnels très différents.

L'objectif de cet article est de proposer un cadre théorique permettant de penser l'hétérogénéité des liens entre grande vitesse ferroviaire et développement économique local dans des pays caractérisés par des niveaux de développement différents. Pour ce faire, nous proposons de dépasser l'analyse de 
l'infrastructure en elle-même, pour nous centrer sur les services qu'elle rend possibles et qui peuvent éventuellement modifier les relations économiques entre les zones desservies. En mobilisant les théories de l'innovation dans les services, nous analysons le transport ferroviaire comme un service et un service ferroviaire à grande vitesse comme un faisceau d'innovations de service (1). Ces innovations sont de nature différente selon les pays et les territoires. Il est alors nécessaire d'enrichir l'analyse de l'innovation de service en intégrant ces différenciations spatiales. En d'autres termes, nous proposons de spatialiser l'analyse en termes de services. Pour ce faire nous mobilisons les analyses institutionnalistes et celles relevant de la sociologie des usages et des systèmes sociotechniques (II).

\section{Le transport ferroviaire: une analyse en termes de services}

Nous proposons de dépasser l'analyse de la seule infrastructure ferroviaire pour conceptualiser le transport ferroviaire comme un service (1.1) et les dessertes ferroviaires à grande vitesse comme un faisceau d'innovations de service (1.2).

\subsection{Le transport ferroviaire, un service coproduit}

Reprenant les travaux de l'innovation dans les services (Gallouj, Weinstein, 1997; De Vries, 2006; Gallouj, Savona, 2009, cf. Gallouj, Djellal, 2015 pour une revue récente), un service de transport ferroviaire est produit à partir de caractéristiques techniques matérielles du train (motrice, système de freinage, capacité des rames), de l'infrastructure (écartement des rails, etc.), de la gare (localisation centrale ou périphérique, mais aussi architecture, aménagement), et du réseau (types de connexion, possibilité d'intermodalité, différents modes ferroviaires), etc., et également de caractéristiques techniques immatérielles (méthodes et procédures permettant à différent trains de circuler sur le réseau, etc.), que l'on peut représenter sous forme d'un vecteur (T) (cf. figure 1).

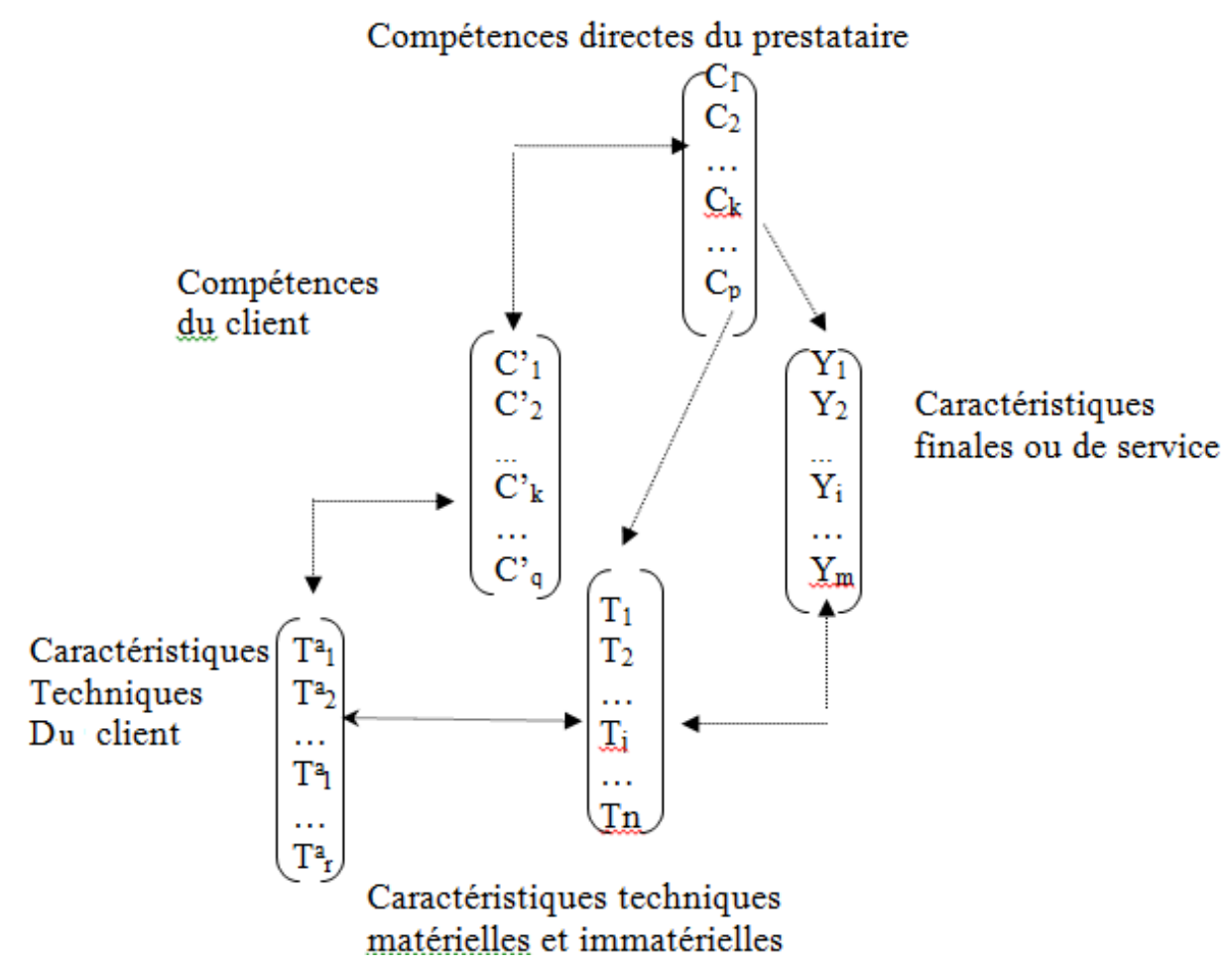

Figure 1.1. La représentation d'un service : élaborée à partir de Gallouj et Weinstein 1997 et De Vries, 2006

Ces caractéristiques techniques ont été construites dans l'histoire au sein de trajectoires technologiques (Dosi, 1982) qui sont en partie spécifiques aux pays, nous y reviendrons. 
Ce service est doté de caractéristiques finales de service (vecteur Y) qui renvoient aux utilités du point de vue de l'usager et qui sont liées au transport en train (vitesse, sécurité, confort ou encore, dans le cas d'une motrice électrique, faible émission de $\mathrm{CO}_{2}$, etc.).

Ce service ferroviaire est fréquemment accompagné d'autres services (restauration dans le train, connexion Wifi, usage de l'électricité, etc.) qui reposent eux-mêmes sur des caractéristiques techniques (prise électrique, etc.). Le transport ferroviaire est ainsi un service central au sein d'un service architectural, i.e. un service qui « est inséré dans un système complexe d'autres services élémentaires qui mobilisent différents types de technologies et de compétences » (Djellal, Gallouj, 2006, p. 1973) (cf. tableau 1.1).

\begin{tabular}{|c|c|c|}
\hline & $\begin{array}{c}\text { Dans le } \\
\text { train }\end{array}$ & En Gare \\
\hline Service de transport & & $\mathrm{x}$ \\
\hline Déplacement d'un lieu à un autre lieu & $\mathrm{x}$ & $\mathrm{x}$ \\
\hline Transport public urbain & & $\mathrm{x}$ \\
\hline Location de voiture, de bicyclette & & $\mathrm{x}$ \\
\hline Taxi & & $\mathrm{x}$ \\
\hline \multicolumn{3}{|l|}{ Aéroport } \\
\hline Parking & & $\mathrm{x}$ \\
\hline \multicolumn{3}{|l|}{ Services associés au transport pendant le déplacement } \\
\hline Accompagnement d'enfants, de personnes en situation de handicap & $\mathrm{x}$ & \\
\hline Service de restauration & $\mathrm{x}$ & \\
\hline Connexion Wifi & $\mathrm{x}$ & \\
\hline Connexion électrique & $\mathrm{x}$ & \\
\hline \multicolumn{3}{|l|}{$\begin{array}{c}\text { Services aux voyageurs (touristes, habitants, professionnels) } \\
\text { avant ou après le déplacement }\end{array}$} \\
\hline Restauration & & $\mathrm{x}$ \\
\hline Hôtels & & $\mathrm{x}$ \\
\hline Journaux & & $\mathrm{x}$ \\
\hline Réservation en ligne $^{1}$ & & $\mathrm{x}$ \\
\hline Retrait d'argent & & $\mathrm{x}$ \\
\hline Connexion Wifi & & $\mathrm{x}$ \\
\hline Mise à disposition de pianos & & $\mathrm{x}$ \\
\hline $\begin{array}{c}\text { Mise à disposition de bicyclettes permettant de recharger des } \\
\text { batteries de terminaux mobiles }\end{array}$ & & $\mathrm{x}$ \\
\hline Mise à disposition de salles de prières & & $\mathrm{x}$ \\
\hline Informations touristiques & & $\mathrm{x}$ \\
\hline Bureaux temporaires & & $\mathrm{x}$ \\
\hline Commerces & & $\mathrm{x}$ \\
\hline \multicolumn{3}{|l|}{ Salon de coiffure, de beauté, masseurs } \\
\hline Services postaux & & \\
\hline
\end{tabular}

\footnotetext{
${ }^{1}$ La réservation en ligne étant évidemment possible dans d'autres lieux.
} 
Tableau 1.1. Quelques services associés au transport ferroviaire

D'autres caractéristiques de service sont liées à la gare et à son aménagement (parking, restauration, vente de journaux, interconnexion avec d'autres modes de transport, location de voitures, distributeur de billets, commerces, bureaux, services postaux, services à la personne, mise à disposition de pianos, de bicyclette pour recharger un téléphone, services religieux, etc.). C'est ainsi une kyrielle de services qui sont aujourd'hui proposés aux usagers des transports ferroviaires comme c'est le cas depuis longtemps dans les gares japonaises. L'adjonction de nouveaux services au service architectural repose sur une logique d'innovation extensive selon la terminologie de Djellal et Gallouj, 2006.

Comme tout service, le service associé à la desserte ferroviaire dans le train ou en gare est coproduit dans la relation entre un ou plusieurs prestataires (privés et/ou publics) et un client. Le service est « un acte, un processus qui s'inscrit dans le temps et dans une relation (de coproduction) entre un client et un prestataire » (Djellal et Gallouj, 2002, p. 135).

Du point de vue de l'offre, le service de déplacement est assuré conjointement par un ou plusieurs prestataires différents (exploitant, gestionnaire de l'infrastructure (entretien des voies et du réseau électrique, tarification et attribution des sillons, etc.), dans certains cas, collectivités publiques, etc.). L'existence de plusieurs prestataires nécessite de les appréhender au sein d'un modèle multi-agents comprenant des acteurs privés, mais également publics (Windrum et Garcia-Goni, 2008) qui doivent se coordonner. Ainsi la restauration à bord est produite par l'opérateur de transport, mais également par l'entreprise de restauration qui dispose du marché. Les services de connexion wifi dans les trains sont produits par l'opérateur ferroviaire et le prestataire de services de téléphone mobile, etc...

De même, dans les gares, le service de restauration est produit par le gestionnaire de la gare et les entreprises de restauration qui y sont localisées, etc...

Ces prestataires disposent de compétences (vecteur C). Reprenant la distinction de Windrum et Garcia-Goni (2008) ces compétences peuvent être des compétences de back office (par exemple, définition d'une grille horaire) ou de front office (par exemple, accueil des voyageurs en gare).

Du point de vue de la demande, les clients peuvent également être de nature diverse (entreprises, ménages ou administrations). Un service de transport peut en effet être utilisé pour des mobilités différenciées (professionnelle, de loisirs et de tourisme, domicile-travail, domicile-études, etc.). Ces clients sont dotés de compétences (vecteur C'), mais également, comme le suggère De Vries (2006), de caractéristiques techniques (vecteur T') qui conditionnent leur capacité à coproduire leur service. Leurs caractéristiques techniques et leurs compétences leur permettent d'interagir avec celles du ou des prestataire(s) non pas directement, mais à l'aide de leur technologie (De Vries, 1986). Par exemple, lorsqu'un client réserve son billet de train sur internet avec son ordinateur personnel, il doit disposer d'un ordinateur (caractéristiques techniques) et de compétences liées à sa capacité à utiliser l'outil informatique pour imprimer les billets, construire son service de transport et rechercher les meilleurs tarifs. Il doit également disposer d'une carte bancaire lui permettant de régler le billet. S'il veut pouvoir disposer d'un e-billet, sans l'imprimer, il doit être équipé d'un Smartphone, etc. Les entreprises ou les administrations peuvent également négocier en partie les tarifs.

Ce modèle de base doit cependant être enrichi pour prendre en compte d'autres acteurs qui, s'ils ne sont pas directement des clients ni des prestataires, influent sur la définition du service: associations d'usagers, chambres de commerce et d'industrie, agences de développement, offices de tourisme, etc.). $\mathrm{Du}$ fait de leur taille, de leur audience, de leur capacité de lobbying et des compétences dont ils disposent, ces derniers peuvent de façon plus ou moins directe peser sur la définition des 
caractéristiques techniques (ouverture de point d'arrêt) ou de services (horaires, fréquences, etc.). Une desserte ferroviaire à grande vitesse correspond alors à différentes innovations.

\subsection{Les innovations associées à la desserte ferroviaire à grande vitesse.}

L'analyse de Gallouj et Weinstein (1997) permet de distinguer six modèles d'innovation dans les services :

- Une innovation radicale qui correspond à la création d'un nouveau système de caractéristiques techniques et de services et de compétences ou, dans une définition extensive, à un nouveau système de caractéristiques et de compétences, mais qui laisse les caractéristiques de service inchangées,

- Une innovation d'amélioration, qui remet en question certaines caractéristiques techniques ou de service, et/ou certaines compétences,

- Une innovation incrémentielle ou innovation par addition ou innovation de substitution, qui correspond à l'ajout d'une ou de plusieurs caractéristiques de services, qui augmentent l'utilité des agents qui en bénéficient, mais qui modifient à la marge la structure générale du système,

- Une innovation de recombinaison, ou architecturale qui recombine ou découpe certaines caractéristiques techniques ou finales, qui associe voire même intègre des " produits-services " existants,

- Une innovation ad hoc, qui est une innovation produite pour résoudre un problème spécifique ou pour un client spécifique,

- Une innovation de formalisation, qui consiste à spécifier des caractéristiques de service, à le rendre moins flou.

Ces modèles permettent de penser une desserte ferroviaire à grande vitesse comme un faisceau d'innovations en termes de caractéristiques techniques, mais également de service.

Premièrement, l'introduction d'une desserte ferroviaire à grande vitesse est à l'origine d'innovations d'amélioration des caractéristiques techniques du train (puissance du moteur, stabilité, etc.) et de la ligne (Campos, De Rus, 2009 pour une analyse des différents types de ligne et de train). Ce type de train circule à grande vitesse (plus de $250 \mathrm{~km} / \mathrm{h}$ ) dans certains pays uniquement sur des lignes spécifiques, dans d'autres parfois aussi sur des lignes existantes.

Deuxièmement, la grande vitesse ferroviaire conduit à une amélioration des caractéristiques de services à plusieurs égards : accroissement de la vitesse commerciale et/ou des fréquences, diminution de la durée des trajets. Des destinations nouvelles peuvent devenir accessibles directement par voie ferroviaire ; des ruptures de charge peuvent être supprimées.

Troisièmement, une desserte ferroviaire à grande vitesse est également une innovation incrémentielle ou incrémentale concernant les caractéristiques de service au sens où elle correspond à l'ajout d'une ou de plusieurs caractéristiques de services. C'est le cas :

1) Lorsque de nouveaux services liés au transport à grande vitesse sont apportés aux voyageurs qui l'utilisent ou lorsque de nouveaux modes de gestion des relations à la clientèle sont mis en place. Par exemple, une connexion Wifi dans les trains est une innovation incrémentielle qui permet de se déplacer à grande vitesse tout en étant simultanément connecté par Wifi. L'e-forfait ou l'e-billet constituent de nouveaux modes de gestion des relations à la clientèle. Ces innovations nécessitent de nouvelles caractéristiques techniques et exigent des changements dans les compétences des prestataires et/ou des clients.

2) L'image associée aux dessertes TGV (cf. Bazin et al., 2011, pour une revue de la littérature sur ce sujet) peut aussi être analysée comme une nouvelle caractéristique de service que nous qualifions de 
sémiotique au sens où elle est porteuse d'un signe de distinction en termes de modernité pour les territoires desservis.

Quatrièmement, la desserte peut se produire dans le cadre d'une innovation architecturale ou de recombinaison. Ainsi l'introduction d'une desserte ferroviaire à grande vitesse peut être le moment pour le gestionnaire de la gare, seul ou en interaction avec d'autres prestataires, d'ajouter des services supplémentaires en gare (magasins, centre de beauté, salle de réunion, bureau, etc.).

Ces services supplémentaires peuvent conduire à modifier le service central : la fréquentation de certaines gares n'est plus liée uniquement au transport ferroviaire, mais au fait qu'elle est un espace de vie (Mannone, 1995). La gare au-delà de la fonction nodale liée au transport, est une place (Bertolini, Spit, 1998). Ainsi des partenariats entre le gestionnaire de la gare et des prestataires de services peuvent être mis en place, élargissant les services disponibles dans les gares. C'est le cas des accords signés par entre Regus, prestataires de location de bureaux à l'heure ou à la journée avec Gare et Connexions en France, avec Thalys en Belgique et Trenitalia en Italie (Pagliara et al., 2016).

De la même façon, le prestataire du service de transport peut proposer un service de transport global qui comprend plusieurs services de transport; des prestataires de différents services de transport se coordonnent pour fournir collectivement un package de services de transport qui va remplacer les différents services fournis jusqu'alors par des prestataires séparés. La production de ces innovations architecturales qui convoquent plusieurs acteurs peut être analysée comme une innovation relationnelle.

Cinquièmement, la production même de la desserte peut donner naissance à des innovations relationnelles (Delaplace, 2012) qui traduisent l'émergence de partenariats, qui jusque-là n'existaient pas, entre des acteurs pour produire un service. La desserte peut, en effet, dans certains cas, être coproduite par les collectivités locales, éventuellement les Etats desservis, voire des acteurs privés dans le cadre de Partenariat Public Privé (PPP). En décidant de financer ex ante les lignes à grande vitesse, les collectivités participent à la définition des caractéristiques techniques sur lesquelles repose la desserte (localisation des arrêts, des gares, etc.). Par ailleurs, les projets de desserte ferroviaire à grande vitesse peuvent produire de la coordination et induire des innovations relationnelles dans les territoires desservis. En effet, ces projets font l'objet de débats auxquels participent les CCI, les agences de développement local, les associations d'usagers, etc.. Ces débats contribuent à façonner les caractéristiques techniques (tracé, la localisation des gares, etc.), les caractéristiques de services (fréquence, horaires) et, dans certains cas, les questions d'interconnexion notamment avec la desserte ferroviaire classique et d'intermodalité. Les associations d'usagers, en particulier en tant que représentantes des contribuables, en faisant valoir que ceux-ci participent au financement de la desserte, peuvent participer à ces débats et peser sur la définition et les modifications ultérieures de la desserte. Ces différentes innovations sont regroupées dans le tableau 1.2. 
Puissance de la motrice

Stabilité des rames à vitesse élevée

Conception et fonctionnement des lignes à grande vitesse

Conception d'une connexion wifi à grande vitesse...

Innovations d'amélioration en termes de caractéristiques de service

Elévation de la vitesse et diminution des temps de trajet

Augmentation des fréquences

Gestion de l'interconnexion

Nouveaux modes de gestion des relations à la clientèle (e-billet, e-forfait)

Suppression de la rupture de charge

Innovations incrémentielles en termes de caractéristiques de service

Connexion Wifi dans le train

Billet combiné (Air/fer, TGV+ Hôtel, TGV + concert, etc.)

Location de bureaux temporaires en gare

Effet d'image

Effet place de la gare...

Innovations relationnelles associées à la production du service

Partenariat Public-privé

Club TGV en France

Partenariat avec les collectivités locales desservies...

Innovations architecturales associées à la production du service

Réaménagement de la gare et de ses abords

Introduction de nouveaux commerces et services en gare...

Tableau 1.2. Principales innovations associées à la grande vitesse ferroviaire

\section{La relation de service associée à une desserte ferroviaire : une relation différenciée dans l'espace}

Si un service s'inscrit dans une logique relationnelle entre un client et un ou des prestataires, ces derniers sont localisés et les caractéristiques techniques tout comme les caractéristiques de services sont inscrites dans des sociétés données (2.1). Le faisceau d'innovations associé à la grande vitesse ferroviaire est alors différencié dans les espaces nationaux et selon les territoires (2.2). Les potentiels de mobilité l'étant également, les interactions entre dessertes ferroviaires à grande vitesse et développement local sont spécifiques à chaque territoire (2.3).

\subsection{Vers une spatialisation de la relation de service et de l'innovation dans les services}

Comme évoqué par Djellal et Gallouj, 2002, si le service est un acte, un processus qui s'inscrit dans le temps, il s'inscrit également dans l'espace. Nous proposons alors la définition suivante : le service est « un acte, un processus qui s'inscrit dans le temps et dans l'espace et dans une relation (de coproduction) entre un client et un prestataire localisés ». Les prestataires comme les clients, les 
caractéristiques techniques comme les caractéristiques de services et les usages sont en effet localisés dans des sociétés données.

Les innovations de services sont alors également localisées. Pour reprendre l'analogie de Madeleine Akrich $(1989$, p. 31) il faut dépasser «la métaphore balistique (qui) suppose l'existence séparée d'un projectile, la technologie, et d'un milieu, la société ». Les évolutions des techniques interagissent avec des évolutions économiques et sociales. Les techniques et l'économie -que ces techniques conditionnent- sont encastrées dans le social (Granovetter, 1985). Leurs évolutions sont favorisées ou entravées par des institutions qu'elles soient informelles (sanctions, coutumes, traditions, etc.) ou formelles (lois, règlements, etc.) (North (1991, p. 97). Ces institutions qui sont le fruit d'une construction historique diffèrent dans le temps et dans l'espace. Certaines relèvent de l'échelle nationale (les lois, la langue, parfois la monnaie, les valeurs, les règles de la concurrence, etc.), d'autres peuvent parfois relever de l'échelle locale ou d'une échelle organisationnelle (la coopération, la confiance, etc.).

Ainsi du côté de l'offre, les caractéristiques techniques et les compétences qui sous-tendent le service reposent sur des technologies données. La technique étant insérée dans l'économie et l'économie dans le social, les caractéristiques techniques sur lesquelles repose la production du service sont inscrites dans une société donnée. De même, les compétences des prestataires, mais également leurs valeurs (intérêt général, satisfaction du client, etc.) dépendent en partie des institutions et des règles de fonctionnement de la société dans laquelle elles s'inscrivent.

Du côté de la demande, et si l'on excepte De Vries, 2006 et l'intégration des caractéristiques techniques des clients (cf. supra), ces clients et les déterminants de leurs comportements -au-delà des technologies qu'ils mobilisent- ne sont que rarement analysés. De même, force est de constater que les analyses du rôle des clients dans l'innovation sont moins nombreuses. Les études de cas sur l'évolution des techniques « ont souvent laissé de côté le problème de l'adoption des technologies par les utilisateurs finals » (Akrich, 1990, p. 83). La littérature sur les systèmes d'innovation s'est en effet pour l'essentiel concentrée sur l'offre (Geels, 2004, p. 900). «Evolutionary economics, business studies and innovation studies tend to focus mainly on the production-side and the creation of knowledge and innovation (e.g. learning within firms, organisational routines, knowledge management), while the user side has received less attention".

Pourtant les usages sont eux aussi déterminés par des valeurs, des règles, i.e. des institutions qui façonnent les préférences des agents (Windrum, Garcia Goni, 2008). Ainsi les caractéristiques de service dépendent des règles qui rendent l'usage du service possible. Pour comprendre les dynamiques d'innovation, l'accent doit ainsi être mis non seulement sur l'innovation, mais aussi sur l'usage et la fonctionnalité (Geels, 2004). Le développement de service de garde d'enfants n'est possible que dans une société qui autorise voire valorise le travail de la femme. L'oenotourisme ne peut se développer dans une société qui prohibe la consommation d'alcool. Tout comme pour l'offre, ces règles, ces valeurs, i.e. ces institutions, sont différentes d'un espace à un autre. Ainsi les actions des agents sont-elles encastrées dans des tissus d'institutions susceptibles d'être différents puisque ces agents sont localisés dans des sociétés données. Ce qui est possible dans un pays ne l'est pas dans un autre ; ce qui est possible dans un territoire peut ne pas l'être dans un autre. L'ensemble des choix possibles offerts à chaque agent est en partie déterminé par sa localisation. En un lieu donné et en un temps $t$, cette localisation se traduit par des contraintes techniques, des règles de comportement, des valeurs, mais aussi des opportunités à la fois pour les prestataires et pour les clients.

Les analyses en termes de système sociotechnique (Geels, 2004) prennent ainsi en compte les systèmes qui englobent et conditionnent le système technique en intégrant la dimension institutionnelle, mais également les acteurs et leurs comportements (figure 2). 


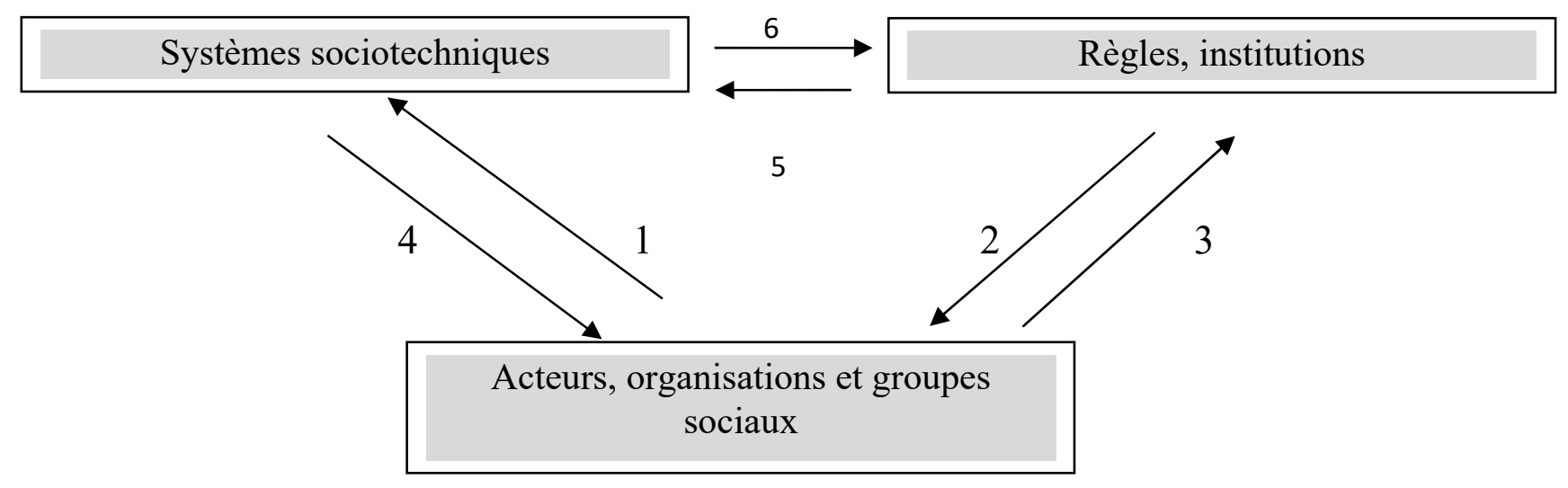

1. Les Systèmes sociotechniques ne fonctionnent pas d'eux-mêmes, mais par l'intermédiaire d'acteurs et d'organisations.

2. Les acteurs agissent dans un contexte de règles qui guident leurs perceptions et leurs (inter)actions.

3. Les acteurs portent et (re) produisent les règles.

4. Les Systèmes sociotechniques, artefacts et conditions matérielles forment le contexte de l'action. Ils rendent possible et contraignent (théorie de l'acteur-réseau).

5. Les règles ne sont pas juste intégrées dans la tête des acteurs, mais aussi dans les artefacts (i.e. les scripts de Latour).

6. Les systèmes sociotechniques, artefacts et conditions matérielles façonnent les règles, les cadres, les standards, etc.. La flexibilité interprétative est contrainte par les possibilités techniques/matérielles

Figure 2. Trois dimensions analytiques inter-reliées (à partir de Geels, 2004, p. 903)

Ainsi, et comme le soulignait déjà Klein dans un article paru en 1998, l'innovation que représente le TGV est une innovation sociotechnique (Klein, 1998).

Il est alors nécessaire d'enrichir l'analyse de l'innovation de services en intégrant les systèmes de valeurs, de règles, i.e. les institutions qui influent à la fois sur les caractéristiques techniques, sur le comportement des agents et sur l'usage et l'appropriation (Proulx et al. 2007) ou la domestication (Haddon, 2011) possible du service. Pour ce faire, nous proposons d'intégrer dans la figure 1 un vecteur I caractérisant ces institutions (figure 3). 


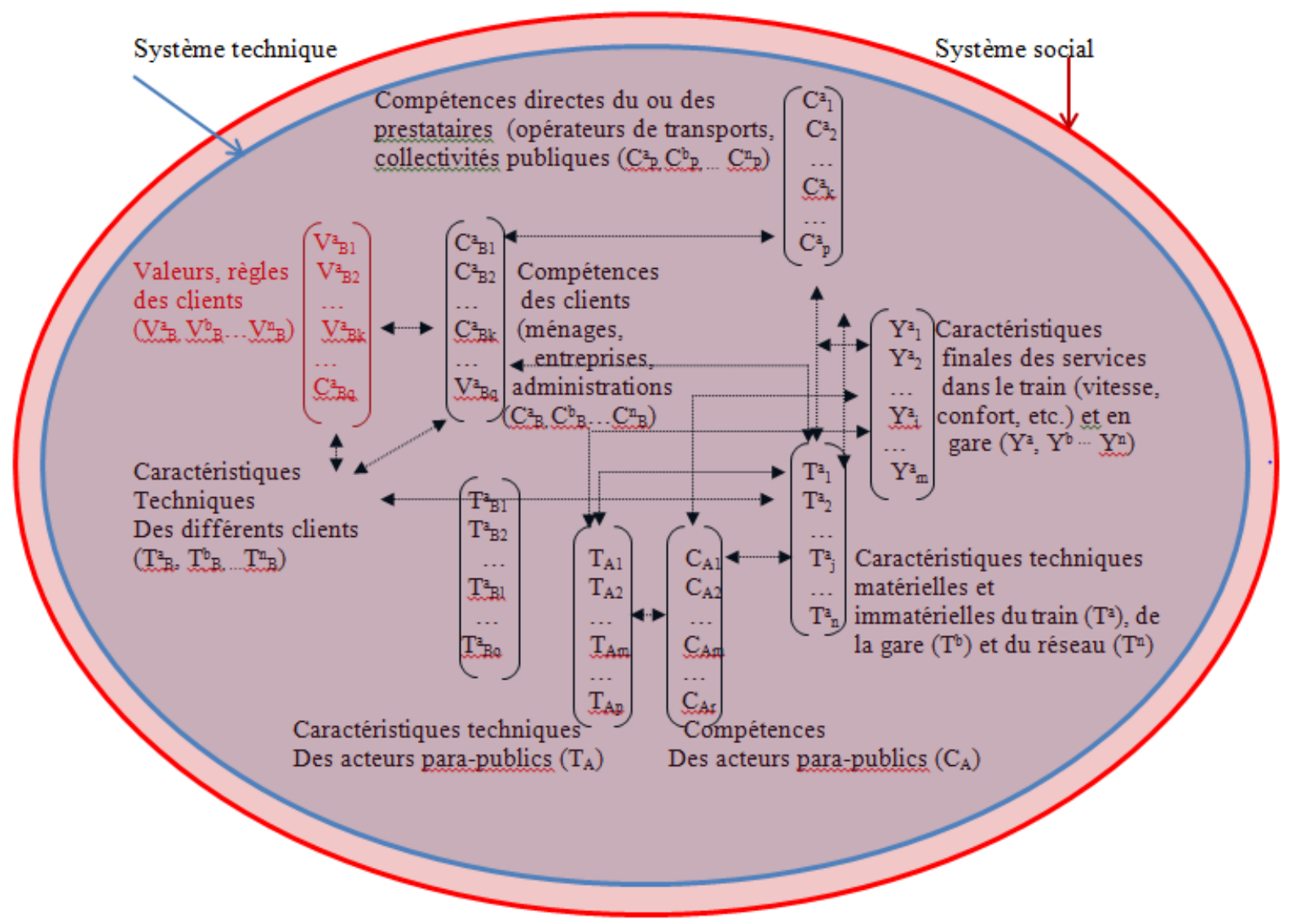

Figure 3. Une nouvelle représentation d'un service intégrant la dimension institutionnelle de la coproduction du service

En outre, il nous semble nécessaire d'élargir le concept de compétences pour caractériser la capacité tant du prestataire que du client à coproduire le service et d'introduire le concept de ressources. Audelà de ses compétences, le prestataire dispose de ressources qui lui sont propres (Wernerfelt, 1984), ou qu'il peut se procurer dans son environnement. Ces ressources qui ont été construites historiquement par celui-ci dans l'interaction avec son environnement peuvent être spécifiques (Colletis et Pecqueur, 2005) au sens où elle n'existent que dans certains lieux (ressources naturelles, patrimoines, etc.). Ainsi la capacité du prestataire de transport à produire un package touristique dans une destination donnée dépendra des ressources touristiques dans cette destination.

De même le client dispose de ressources (revenus, compétences, connaissances, etc.) qui lui permettent ou non de coproduire le service. Sans revenus suffisants, le client ne pourra acheter son billet. Les ressources culturelles (ou capital culturel) dont il dispose conditionneront sa capacité à envisager telle ou telle destination.

Ainsi nous substituons le vecteur de ressources $\mathrm{R}$ au vecteur de compétences $\mathrm{C}$ tant du côté des clients que du côté des prestataires (figure 3).

Cette prise en compte du caractère institutionnel de la technique et de la dimension spatialisée de la relation de services permet de comprendre les raisons pour lesquelles une desserte ferroviaire ne peut être analysée comme ayant automatiquement des impacts sur un territoire : le service de déplacement qui lui est associé est en effet coproduit par les agents localisés sur les territoires que cette desserte ferroviaire permet de connecter. Le service en acte est territorialisé ${ }^{2}$.

\footnotetext{
${ }^{2}$ La dimension sociale et institutionnelle de la relation de services est cependant plus complexe que celle retranscrite dans le schéma ci-dessous. En effet, prestataires et clients ne sont pas nécessairement insérés dans le même système sociotechnique. Un prestataire relevant d'un pays peut coproduire un service avec un client localisé dans un autre pays.
} 


\subsection{La grande vitesse ferroviaire: des innovations en termes de caractéristiques techniques et de service différentes selon les territoires}

Les innovations en termes de caractéristiques techniques dont les agents localisés peuvent bénéficier sont différentes selon les pays et parfois selon les territoires (2.2.1). Il en va de même des innovations en termes de caractéristiques de service (2.2.2).

\subsubsection{Des innovations en termes de caractéristiques techniques différentes selon les pays}

Les innovations d'amélioration des caractéristiques techniques sont d'importance variable selon les pays puisque le type de réseau et de train ainsi que la localisation de la gare sont susceptibles d'y être différenciés. Ainsi les caractéristiques techniques du train à grande vitesse varient selon les pays (Givoni, 2006). Celui-ci n'est parfois qu'une adaptation des techniques ferroviaires préexistantes qui s'inscrit dans la trajectoire technologique du ferroviaire (France, Espagne, Japon). Dans d'autres pays, il correspond à des innovations des caractéristiques techniques radicales comme le pendulaire, en Espagne pour l'Alaris, le Pendolino en Italie, ou le train à sustentation électromagnétique dans certains territoires du Japon ou encore le Transrapid reliant l'aéroport Pudong à Shanghai. Dans certains pays, plusieurs technologies différentes coexistent (Italie, Japon, Espagne, etc.). En outre, le type et la forme de réseau sur lesquels circulent les trains à grande vitesse ainsi que leurs fonctionnalités sont différents selon les pays (Campos, Rus de, 2009; Givoni, 2006 ; Vickerman, 1997) : voies dédiées (Japon), circulation sur voies dédiées et sur réseau conventionnel (Campos, Rus de, 2009) (France, Allemagne, Italie, et dans le futur, Maroc). En Espagne, les trains à grande vitesse AVE ne peuvent circuler sur le réseau classique en raison d'un écartement des rails différent. En France, la desserte sur ligne classique s'inscrit dans un contexte institutionnel spécifique caractérisé par la mise en œuvre des premières lois de décentralisation. Cette desserte sur ligne classique permet en effet de limiter les coûts liés à la construction d'une ligne à grande vitesse tout en desservant un nombre important de territoires ; chaque territoire souhaitant son TGV.

La forme du réseau est aussi directement liée à des considérations institutionnelles et à la hiérarchie urbaine de chaque pays. La structure du réseau est ainsi différente: alors que le réseau est centré sur une grande ville, Paris, en France, Madrid en Espagne ou Séoul en Corée, en Allemagne en revanche il permet de desservir de nombreuses villes ; desserte qui est associée au caractère fédéral de ce pays. La localisation de la gare est également différente selon les pays (Givoni, 2006, Urena et al., 2009): nouvelles gares construites spécifiquement parfois en rase campagne (gare Haute Picardie, gare Meuse en France, etc.), parfois à proximité de villes (gare Champagne-Ardenne, etc.), parfois connectées au réseau ferroviaire classique, ou anciennes gares centrales rénovées. En France la localisation des gares est le fruit de compromis institutionnalisé entre la SNCF qui souhaite des gares excentrées permettant d'optimiser les gains de temps et le souhait des élus d'obtenir une desserte au cœur de leurs villes. Les réseaux techniques sont aussi des réseaux territoriaux. Ce " ne sont pas que des matériaux et des dispositifs techniques mis en place dans l'espace : ils sont aussi et surtout le fruit de décisions humaines, sociales, politiques ; le résultat d'une longue négociation entre les différents acteurs sociaux »(Bassand, Galland, 1993, cité par Fachinetti-Mannone, 2016).

Ainsi les caractéristiques techniques liées à la grande vitesse ferroviaire sont en partie spécifiques aux pays et parfois également à l'échelle infranationale. Ces différences doivent être prises en considération dans l'analyse des interactions entre grande vitesse ferroviaire et développement économique local puisqu'elles affectent l'utilisation de l'infrastructure (Campos, Rus de, 2009), et par conséquent les services de déplacement possibles, l'existence ou non d'interconnexion en termes de transport, mais également les usages de la gare et du foncier qui l'entoure.

\subsubsection{Les innovations en termes de caractéristiques de service}

Les innovations en termes de caractéristiques de services sont-elles aussi différenciées selon les pays et les territoires. 
Premièrement, si le transport ferroviaire est dit à grande vitesse lorsqu'il atteint $250 \mathrm{~km} / \mathrm{h}$ en vitesse de croisière, l'accroissement de la vitesse est plus ou moins important selon les territoires desservis en fonction de leur topographie, de leurs caractéristiques géographiques (Campos, Rus de, 2009), de leur caractère plus ou moins urbanisé, etc... D'autres innovations en termes de caractéristiques de service telles que la fréquence, son cadencement, les interconnexions ferroviaires et les différentes formes d'intermodalité (quand elles ont été créées) sont aussi différenciées. Ces innovations dépendent des caractéristiques techniques (type de train et de ligne utilisé, cf. supra), mais également des choix faits localement. De même, les destinations envisageables sont susceptibles d'être différenciées. Ainsi si en France les dessertes vers Paris existent de façon quasi-systématique (le réseau français étant centré sur la capitale), en revanche, seules les villes disposant de gare d'interconnexion sur le réseau à grande vitesse peuvent bénéficier d'une connexion directe avec d'autres villes de province et l'international. En Allemagne, « la recherche de la vitesse pure n'est pas la priorité » (Woessner, 2014); l'essentiel est de desservir une majorité des villes constituant l'armature urbaine des länders. Le caractère institutionnalisé de la desserte ferroviaire à grande vitesse apparaît de nouveau clairement.

Deuxièmement, l'ampleur de l'innovation dépend également de la qualité initiale du service ferroviaire (Garmendia et al., 2008), de la stratégie de l'opérateur de transport, de la concurrence (au sein du mode ferroviaire et avec d'autres modes) et des stratégies des principaux concurrents. De nouveau, ce sont des éléments qui sont différenciés dans le temps et dans l'espace, à l'échelle nationale, mais également locale. Dans certains pays le réseau ferroviaire est dense et couvre l'ensemble du territoire (France), dans d'autres il est presque totalement absent (Etats-Unis). A l'intérieur d'un même pays, certaines villes bénéficiaient d'une desserte ferroviaire importante depuis longtemps, d'autres en étaient à l'écart. Dans les pays en développement, l'innovation de services est d'autant plus importante que le réseau est de mauvaise qualité ou inexistant.

Troisièmement, les innovations incrémentielles sont également différenciées entre pays et au sein d'un même pays, selon les territoires. Ainsi l'effet d'image associée à la desserte peut varier à l'échelle nationale en fonction des projets de rénovation et/ou de construction de gares nouvelles, mais également des stratégies locales de communication. La construction des gares nouvelles repose parfois sur des investissements importants en termes d'image. Des concours d'architecture sont lancés (Gare de Liège-Guillemin en Belgique, Gares TGV du Maroc, etc.), un marquage local de la gare parfois effectué (Vignes pour la Gare Champagne-Ardenne, Horloge monumentale à la nouvelle gare TGV de Besançon-Franche-Comté). L'objectif est ainsi de spécifier la gare en fonction de son territoire d'accueil.

Dans les pays en développement, cet effet d'image peut être extrêmement important. Ainsi les gares de Tanger ou de Kénitra au Maroc pour ne citer qu'elles (cf. figures 4 et 5) conduisent à l'irruption d'une hyper modernité dans un pays encore marqué par un niveau de développement faible ${ }^{3}$.

\footnotetext{
${ }^{3}$ Avec 7790 \$ de PIB par habitant (\$ PPA internationaux courants) en 2014, le Maroc fait partie des pays à revenu moyen inférieur, selon la Banque Mondiale. En termes d'IDH, il est classé en $126^{\text {ème }}$ position sur 188 pays en 2015. 


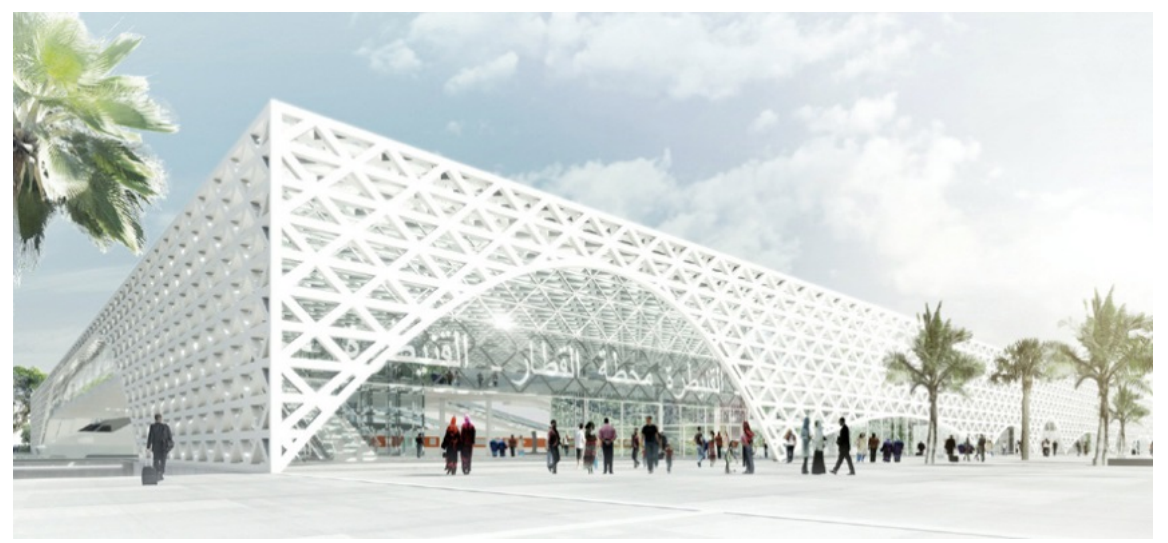

Figure 4. Gare de Kénitra (ONCF)

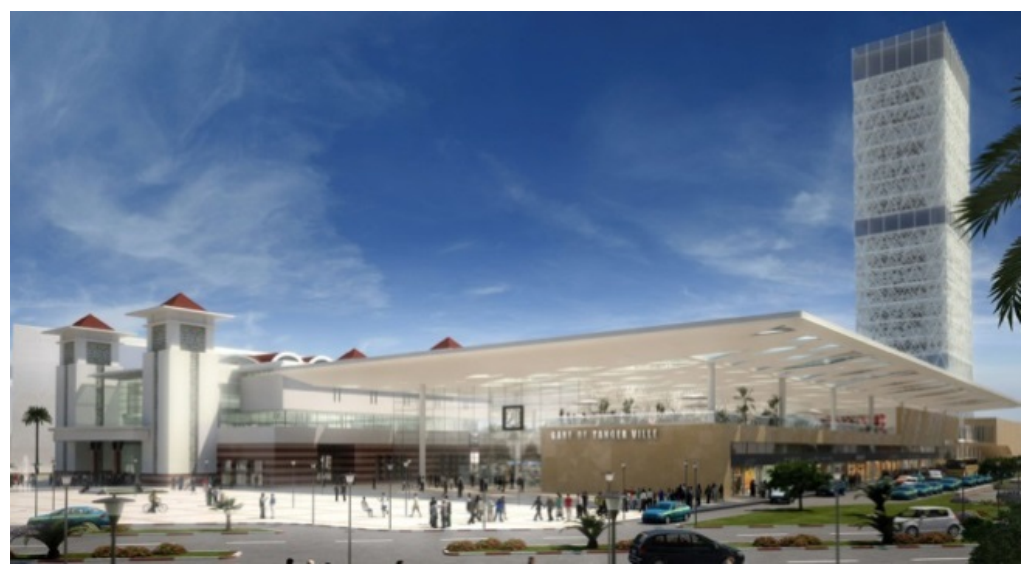

Figure 5. Gare de Tanger Ville (ONCF)

Si la gare peut faire l'objet d'un processus de spécification, c'est également le cas du train et du service de transport qu'il autorise. Ainsi au Japon, le Shinkansen "Toreiyu" vers Yamagata, met à disposition des voyageurs un spa pour les pieds et leur propose de déguster des plateaux-repas avec des spécialités de cette province réputées pour la qualité de ses fruits. Le train, sa décoration intérieure avec des tablettes en bois de cerisier, des voitures ornées d'accessoires en fer forgé représentant des fruits, etc.,- et le service à bord sont alors spécifiés en fonction du territoire qu'il dessert. De la même façon en France au moment de certains festivals (Eurockéennes de Belfort, Francofolies de la Rochelle, Flâneries musicales de Reims, etc.), la SNCF propose des places dans des TGV Live associant services de transport et concert.

D'autres innovations incrémentielles sont parfois disponibles uniquement sur certaines dessertes et /ou dans certains pays. C'est le cas en France de la connexion wifi, de l'e-forfait ${ }^{4}$, etc.. Les services fournis dans les trains sont différenciés selon les pays (ligne de sonorisation, cinéma à bord, services de presse, etc. $)^{5}$.

Enfin les innovations extensives associées au service architectural qui consistent à accroître la diversité des services proposés en gare (services à la personne, services d'interconnexion, voire services religieux, etc.) dépendent des stratégies des prestataires qui, là aussi, sont variables selon les pays et les territoires en fonction des valeurs qui les animent et des ressources dont ils disposent. Les services dans la gare TGV La Part Dieu sont évidemment sans commune mesure avec ceux de la gare

\footnotetext{
${ }^{4}$ L'e-forfait a ainsi été mis en place à Reims exclusivement pour les abonnés empruntant la liaison Reims-Paris-Est. Les connexions Wifi à $320 \mathrm{~km} /$ heure sont disponibles dans les TGV Est-Européens. Une connexion Wifi à des vitesses moindres a également été testée auparavant sur le Thalys et sur le TGV Paris-Bordeaux-Pau.

${ }^{5} \mathrm{C}$ 'est le cas en Allemagne où une ligne de sonorisation pour chaque voyageur est proposée dans les ICE. Dans les AVE-S100 sur les trajets Barcelone-Paris, un service de cinéma à bord est proposé ; dans les Thalys ce sont des services de presse multilingue, etc.. (c) 2017 ISTE OpenScience - Published by ISTE Ltd. London, UK - openscience.fr 
Meuse en France. Au Maroc, les gares à grande vitesse accueillent outre des commerces, une salle de prière, etc.

Quatrièmement, les innovations relationnelles associées à la production de la desserte sont aussi différentes dans l'espace puisque l'organisation du transport ferroviaire l'est (séparation éventuelle entre le gestionnaire de l'infrastructure et le transporteur exploitant, partenariats public-privé, etc.). Ainsi en France, de 1997 jusque 2014, RFF assurait la gestion de l'infrastructure (entretien des voies et du réseau électrique, tarification et attribution des sillons, etc.) ; la SNCF en tant que transporteur exploitant gérait le matériel roulant et définissait le service de déplacement. La création de SNCF réseau au 1er janvier 2015 a modifié cette organisation. Toujours en France, la collectivité publique veille à ce que soit assuré le droit au transport prévu par la Loti. Depuis 2002, la région est l'autorité organisatrice des transports régionaux. Cette organisation n'est pas la même dans d'autres pays. Par exemple, en Allemagne comme en Italie, il n'y a pas de séparation institutionnelle, mais uniquement une séparation en deux filiales au sein d'une même holding. En Espagne, la séparation institutionnelle a été opérée en 2005 , date à laquelle la gestion de l'infrastructure a été confiée à l'ADIF ; la RENFE n'étant plus que l'exploitant. Ce sont de nouveau des considérations institutionnelles qui conduisent à cette différenciation et qui influent sur les innovations associées à la desserte.

Mais les innovations associées à la desserte ferroviaire à grande vitesse dépendent également des clients qui vont coproduire leur service de déplacement en fonction de leurs potentiels de mobilité. Or ces potentiels peuvent être différents selon les pays et, au sein d'un même pays, selon les territoires. Ces usages sont en effet inscrits là aussi dans le temps et dans l'espace.

\subsection{Potentiel de mobilité et usages de la desserte : des différenciations territoriales marquées}

Les potentiels de mobilité et par conséquent les usages d'une desserte à grande vitesse dépendent du contexte spatio-temporel national dans lequel est inséré le territoire. Ce contexte spatio-temporel influe sur le comportement des agents qui y sont localisés, sur les valeurs et les règles qui les régissent et les ressources dont ils disposent (2.3.1). Mais les usages possibles dépendent également des territoires qui seront desservis, de leurs caractéristiques, des usagers qui y résident de façon permanente ou temporaire et de la stratégie du prestataire sur ces territoires (2.3.2).

\subsubsection{Contexte spatio-temporel national, potentiel de mobilité et usage de la desserte}

Si le contexte spatio-temporel national influe sur les caractéristiques de la desserte ferroviaire (train, vitesse, fréquence, destination, etc.) à laquelle les usagers ont accès (cf. supra), il influe également sur l'usage de cette desserte, i.e. le service de déplacement en acte.

Premièrement, la conjoncture qui varie dans le temps et dans l'espace influence les comportements de mobilité comme l'ont montré Klein et Claisse (1997) au début des années 1990 en France. Nash souligne ainsi que « the perspectives of success of the railroad High-Speed depend widely on the future economic growth » (Nash, 2009, p. 24).

Au-delà de la mobilité, cette conjoncture affecte les anticipations et les investissements des agents privés, mais également les politiques publiques comme en témoignent les difficultés à la fin des années 2000 à remplir le parc d'activités de Bezannes à côté de la gare d'interconnexion Champagne-Ardenne, ou les difficultés de commercialisation des programmes d'immobilier de bureau sur le centre d'affaires Novaxis au Mans en 1993. De même, en Espagne, Valdeluz, près de Guadalajara, est aujourd'hui encore une ville fantôme. Compte tenu de la crise et de ses répercussions immobilières, le projet immobilier autour de la gare n'a pas rencontré le succès escompté (Bozonnet, 2008, Mohino et al., 2014).

Deuxièmement, les potentiels de mobilité sont conditionnés par le contexte institutionnel national. Ainsi, par exemple, la mobilité touristique dépend du temps de travail et du nombre de jours de congés 
payés qui est variable selon les pays. Une réduction du temps de travail, comme ce fut le cas en France jusqu'au début des années 2000 , a accru les potentiels de mobilité et a généré une croissance des séjours touristiques de courte durée6 (les city-breaks) que la desserte TGV a également favorisé. L'idée même de mobilité touristique est différente selon les pays. En Corée du Sud, en raison du faible nombre de jours de congés payés (15 jours), les déplacements sont le plus souvent dévolus aux évènements familiaux. Les potentiels de mobilité sont de fait plus restreints pour les motifs de loisirs et de tourisme.

Les potentiels de mobilité dépendent également des niveaux de revenus qui sont aussi variables selon les pays. Par exemple si les études tendent à montrer qu'il y a relativement peu de différences de mobilité quotidienne en France selon les revenus (Paulo, 2007), ce n'est pas le cas de la mobilité de fin de semaine. Le nombre de déplacements le week-end est supérieur de $2 / 3$ pour le dernier quintile par rapport au premier quintile. La distance parcourue est aussi 2,4 fois plus élevée. De surcroît les mobilités à longue distance (plus de $100 \mathrm{~km}$ ) sont encore plus corrélées aux revenus. A l'échelle internationale les mobilités de longue distance dans les pays en développement sont en retrait par rapport aux pays industrialisés. En outre, selon Doyle et Nathan, (2001), les $20 \%$ les plus riches voyagent 3,5 fois plus loin que les $20 \%$ plus pauvres. Enfin si dans les sociétés occidentales on peut considérer que « les individus habitent dans un lieu, travaillent dans un autre, se distraient dans un troisième » (Paulo, 2007), c'est vraisemblablement moins marqué dans les pays en développement. Dans les pays en développement, ce sont par exemple de façon très importante, les populations les plus aisées qui peuvent voyager à des fins de tourisme7. Le tourisme y est souvent un tourisme international ou le fait d'une minorité locale. Le tourisme ne se répand dans les classes moyennes qu'avec l'élévation du niveau de vie (Cazes, 1983, cité par Berriane, 1993). Les mobilités s'accroissent avec le niveau de développement.

Les potentiels de mobilité susceptibles de générer des usages importants de la desserte dépendent enfin de l'importance accordée au temps et donc à la vitesse. L'usage de la grande vitesse ne peut se comprendre dans une société dans laquelle on n'accorde que peu de valeur au temps. Or chaque individu ne valorise pas ou ne déprécie pas le futur seulement en fonction de son âge, de sa situation, de sa position. «Les conceptions vis-à-vis du temps dépendent des règles du jeu social ; derrière l'échange, il y a communication de signes et de symboles. Elles dépendent également de la place qu'occupent les agents dans la société » (Hugon, 1991, p. 343).

Se déplacer fréquemment sur longue distance est parfois un signe de distinction de même que la grande vitesse est fondamentalement un signe de modernité.

Le service coproduit sur la base des interactions entre prestataires et clients dépendra alors des capacités des agents sur les territoires à utiliser la desserte, en termes monétaires, mais également en termes symboliques.

Ainsi les usages possibles de la desserte sont différenciés selon les pays. Mais, dans un pays et à un moment donné, ces potentialités sont également conditionnées par les caractéristiques intrinsèques des territoires desservis et la stratégie du prestataire sur ces territoires.

\subsubsection{Caractéristiques des territoires, potentialités d'usage et stratégie du prestataire}

\subsubsection{Stratégie du prestataire et potentialités d'usage}

La stratégie du prestataire influe sur les potentialités d'usage. Le ou les prestataire(s) oriente(nt) en effet les potentiels de mobilité en fonction de leur politique tarifaire et du surcoût par rapport à un billet

\footnotetext{
${ }^{6}$ Ainsi entre 1998 et 2004, le nombre de courts séjours a crû de $16,3 \%$; il passe ainsi de 52,2 \% à 55,7 \% de l'ensemble des voyages effectués par les Français (direction du tourisme, 2005).

${ }^{7}$ Certains auteurs considèrent néanmoins que le tourisme interne ne doit pas être sous-estimé dans les pays en développement (Opperman, 1991, Bérriane, 1993). 
sur ligne ferroviaire classique quand cette dernière existe. Or cette stratégie est variable selon les territoires. Ce faisant, le prestataire conditionne les possibilités de coproduction d'un service de déplacement.

L'élévation du prix du billet associée à la grande vitesse ferroviaire est plus ou moins importante selon les pays et, en leur sein, selon les territoires. Ainsi le prix du service de transport TGV Paris Lille est-il le plus onéreux de France au kilomètre, les dessertes Ouigo en revanche - initialement au départ et à l'arrivée de Chessy-Marne-la-Vallée puis dorénavant étendues à Massy TGV vers certaines destinations de l'ouest et du Nord de la France - permettent de voyager à des coûts réduits (Delaplace, Dobruszkes, 2015). Par ailleurs, des billets à prix réduit de façon pérenne ou lors du lancement de la desserte peuvent affecter les usages de la desserte voire influer sur les choix de destination des touristes (Delaplace et al. 2014, 2016, Pagliara et al. 2015).

Le ou les prestataire(s) peuvent construire une grille horaire visant à privilégier certains types de mobilité (migrations alternantes, mobilité professionnelle, mobilité de tourisme, etc.) dans certains territoires et dédier ainsi la desserte à certains usages. De même, ils peuvent proposer des offres supplémentaires, affréter davantage de rames lors d'évènements exceptionnels dans les villes. Ils peuvent également proposer des modalités spécifiques de réservation et d'abonnement (e-forfait par exemple) sur certains trajets, de façon à développer les mobilités.

Les potentialités d'usage d'une desserte dépendent également des caractéristiques des territoires desservis et des usagers qui y résident et/ou y travaillent de façon permanente ou temporaire.

\subsubsection{Caractéristiques intrinsèques des territoires et usages possibles de la desserte}

La desserte est spécifique à chaque territoire. C'est le cas d'une part, du fait des multiples configurations en termes de localisation de la gare selon les pays et au sein d'un même pays (cf. supra). Or selon que la gare est localisée en centre-ville ou en périphérie, les potentiels de mobilité et l'usage de la desserte peuvent être différenciés. Par conséquent la nature du service de déplacement coproduit sera différente.

Ces potentialités d'usage de la desserte sont liées, d'autre part, à l'attractivité respective des territoires nouvellement reliés ou dont la desserte est améliorée et ce, pour les différents motifs de déplacement (travail, professionnel, tourisme, études). Cette attractivité dépend des caractéristiques intrinsèques de ces territoires et notamment leurs ressources qui résultent elles-mêmes de leur géographie et de leur histoire (climat, aménités, patrimoine, etc.). Ces caractéristiques influent sur leur capacité respective à émettre des flux de voyageurs les uns en direction des autres.

Les conditions géographiques et naturelles d'un territoire font que celui-ci dispose de caractéristiques spatiales spécifiques (sa localisation, sa géographie physique, etc.). Ainsi, une localisation près de la montagne ou de la mer, i.e. des destinations propices au développement du tourisme, peut induire des potentialités d'usage accrues de la desserte à des fins de déplacements touristiques.

La localisation d'un territoire par rapport à d'autres territoires et par conséquent les possibilités de gains de temps et de distance entre les territoires desservis (Givoni, 2006; Kamel, Matthewman, 2008 ), la possible proximité de métropoles (Urena et al., 2009) et plus généralement la configuration spatiale et la structure urbaine du pays influent également sur les potentialités d'usage. Ainsi, l'arrivée d'une desserte à grande vitesse peut accroître l'influence de plus grosses villes sur des villes de plus petite taille.

L'histoire d'un territoire, l'existence de patrimoines architectural, culturel influent sur les possibilités de développer des formes de tourisme. Dans certains cas, des tourismes urbains et

\footnotetext{
${ }^{8}$ Sur ce sujet, la littérature est cependant assez partagée. (cf. Bazin et al., 2011, pour une revue de la littérature).

(c) 2017 ISTE OpenScience - Published by ISTE Ltd. London, UK - openscience.fr 
d'affaires qui sont des tourismes de court séjour peuvent être facilités par l'existence d'une desserte TGV de qualité comme en atteste l'analyse de certaines expériences passées en France (cf. Bazin et al., 2011). C'est particulièrement le cas lorsque la gare est encastrée dans un tissu urbain doté de patrimoines eux-mêmes concentrés à proximité.

Enfin, la population (structure des emplois par Professions et Catégories Socio-professionnelles), la nature des entreprises (spécialisation sectorielle, taux de dépendance, etc.) sur le territoire et dans les territoires nouvellement reliés vont affecter les usages de la desserte. C'est le cas plus précisément :

- du type de ménages sur le territoire, qui sont des clients potentiels pour le service ferroviaire, du type de mobilité (professionnelle, migrations alternantes, mobilité touristique, etc.) qui les caractérise et de la valeur qu'ils accordent au temps,

- de la qualification de cette population, et notamment de la présence de cadres. Certaines catégories d'utilisateurs (PCS supérieures) et de voyageurs d'affaires utilisent davantage la desserte à grande vitesse (Klein et Claisse, 1997).

- de la spécialisation sectorielle (industrielle, commerciale, servicielle, etc.) et du type d'entreprises localisées au sein du territoire (grandes ou petites firmes, filiales, type de marchés qu'elles desservent (local, national, international), etc.). Si le territoire est caractérisé par des firmes dont les employés se déplacent fréquemment, une desserte TGV adéquate peut être utilisée pour les déplacements professionnels et inversement. Certaines entreprises ont ainsi une capacité plus importante à utiliser la desserte.

De nouveau, certains de ces éléments sont différenciés selon les territoires. Conformément à la théorie des places centrales, les services «supérieurs » se localisent de préférence dans les grandes villes conduisant à leur concentration spatiale dans ces villes (Mérenne-Schoumaker, Moyart, 2007). Enfin, les potentialités d'usage dépendent de la structure des migrations alternantes entre les territoires nouvellement reliés par desserte ferroviaire à grande vitesse, structure qui favorise en général les plus grandes villes.

De ces potentialités d'usage découlera la possible appropriation de la grande vitesse ferroviaire Cette dernière est en effet différente du simple usage dans la mesure où elle induit une modification des comportements. Il s'agit non seulement d'intégrer l'usage dans sa vie quotidienne, mais également que cet usage « fasse émerger de la nouveauté dans la vie de l'usager. » (Proulx et al., 2007, p. 9-10).

La desserte peut enfin être utilisée par les acteurs locaux comme un outil au service du développement de leur territoire comme en témoignent les nombreux parcs d'activités et quartiers d'affaires qui ont fleuri autour des gares TGV en France, mais également les politiques de communication auxquelles elle est associée.

Ainsi la relation de service associée à une desserte ferroviaire est spécifique dans le temps et dans l'espace. Le service est alors lui-même spécifique à un territoire donné. La prise en compte du caractère institutionnel de la technique et de la dimension localisée de la relation de services permet de comprendre les raisons pour lesquelles une desserte ferroviaire ne peut être analysée comme ayant des impacts sur un territoire : le service de déplacement qui lui est associé est en effet coproduit par les agents localisés sur les territoires que cette desserte ferroviaire permet de connecter. Le service en acte est territorialisé au sens où il est spécifique à un territoire donné.

\section{Conclusion}

Les liens entre dessertes ferroviaires à grande vitesse et développement économique local sont différenciés dans l'espace. Dans cet article, nous avons proposé une conceptualisation de cette hétérogénéité. Tout d'abord, en mobilisant les analyses de l'innovation dans les services, nous avons montré qu'il était nécessaire de dépasser les analyses centrées sur les infrastructures et de se focaliser 
sur les innovations de service induites par une desserte ferroviaire à grande vitesse. Puis nous avons mis en évidence que ces innovations étaient différenciées dans le temps et dans l'espace. Par ailleurs, nous avons souligné qu'il était nécessaire de tenir compte des systèmes de valeurs, des règles, i.e. des institutions qui influent à la fois sur les caractéristiques techniques, sur le comportement des agents et sur l'usage et leur appropriation des innovations de services associées à cette grande vitesse ferroviaire. Cette analyse nous a ainsi conduits à enrichir l'analyse de l'innovation dans les services en proposant une spatialisation de la relation de service via l'intégration dans le modèle initial de deux éléments différenciés dans l'espace, les institutions et les ressources.

Une desserte ferroviaire à grande vitesse ne peut donc être analysée comme ayant automatiquement des impacts sur un territoire. C'est dans la possible réalisation d'une coproduction de services que résident les modifications des comportements et les transformations des territoires desservis. Mais audelà de la question des interactions entre grande vitesse ferroviaire et développement local, c'est donc plus généralement une spatialisation de l'innovation dans les services que cet article propose. Tout comme les agents économiques ne sont pas en apesanteur, les services qu'ils coproduisent sont ancrés dans des territoires dont le fonctionnement est en partie lié aux sociétés dans lesquelles ils s'insèrent.

\section{Bibliographie}

Akrich M. 1989. La construction d'un système sociotechnique : Esquisse pour une anthropologie des techniques. Anthropologie et sociétés, Département d'anthropologie, Université De Laval, 13 (2) : 31-54.

Akrich M. 1990. De la sociologie des techniques à une sociologie des usages ; L'impossible intégration du magnétoscope dans les réseaux câblés de première génération? Techniques et Culture, $\mathrm{n}^{\circ} 16: 83-110$.

Bazin S, Beckerich C, Blanquart C, Delaplace M., Vandenbossche L. 2011. Grande vitesse ferroviaire et développement économique local : une revue de la littérature, Recherche, Transports et Sécurité, $27: 215-38$.

Berriane M. 1993. Le tourisme des nationaux au Maroc (une nouvelle approche du tourisme dans les pays en développement), Annales de Géographie, 102, (570) : 131-161.

Bertolini L., Spit T. 1998. Cities on rails: The redevelopment of railway stations areas, London, E \& FN Spon.

Bozonnet J-J. 2008. "Bienvenidos" à Valdeluz, ville fantôme. Le Monde, 22/10.

Campos J., Rus G. de 2009. Some stylized facts about High-Speed Rail: A review of HSR experiences around the world. Transport policy, 16, pp.19-28.

Colletis G. et Pecqueur B. 2005. Révélation de ressources spécifiques et coordination située, Économie et institutions, [En ligne], 6-7| p. 51-74, mis en ligne le 31 janvier 2013.

Delaplace M., Dobruszkes F. 2015. From low-cost airlines to low-cost high-speed rail? The French case, Transport Policy, 38: 73-85

Delaplace M., Pagliara F., la Pietra A. 2016. Does High-Speed rail affect destination choice for tourism purpose? Disneyland Paris and Futuroscope case studies? Belgeo, 3, en ligne.

Delaplace M., Pagliara F., Perrin J., Mermet. S. 2014. Can High Speed Rail foster the choice of destination for tourism purpose?, Procedia - Social and Behavioral Sciences, EWGT2013 - 16th Meeting of the EURO Working Group on Transportation, 11 (1): $166-175$.

Delaplace M 2012. Pourquoi les « effets » TGV sont-ils différents selon les territoires ? L'hétérogénéité au cœur du triptyque « Innovations, Territoires et Stratégies », Recherche Transports et Sécurité, 28 : 290-302

Djellal F., Gallouj F. 2006. Un modèle d'analyse de la dynamique de l'innovation dans les services : Le cas des services de types architecturaux, Economies et Sociétés, Série EGS, 7 : 1973-2010.

Djellal F., Gallouj F. 2002. A propos de la nature de l'innovation dans les services : les enseignements d'une enquête postale. In Djellal F, Gallouj F (eds.) Technologies et innovations dans la nouvelle économie des services. L'Harmattan, Collection “ Entreprise et Innovation ”, Paris: 135-163.

Dosi G. 1982. Technological paradigms and technological trajectories, Research Policy, 1: 147-162.

Doyle J., Nathan M. 2001. The hypermobile must not be allowed to rule roost, The Guardian, https://www.theguardian.com/business/2001/apr/23/7. 
Fachinetti-Mannone V. 2016. La territorialisation des gares de la grande vitesse, Université de Bourgogne, Habilitation à diriger les recherches soutenue à Dijon le 14 mars

Gallouj F., Weinstein O. 1997. Innovation in services. Research Policy, 26: 537-56.

Gallouj F., Savona M. 2009. Innovation in services: a review of the debate and a research agenda. Journal of Evolutionary Economics, 19: 149-72.

Gallouj F., Djellal F. 2015. Introduction to "services and innovation”, Edward Elgar Publishing .

Geels F.W. 2004. From sectoral systems of innovation to socio-technical systems; Insights about dynamics and change from sociology and institutional theory, Research Policy, 33: 897-920.

Givoni M. 2006. Development and impact of the modern high-speed train: a review, Transport reviews, 26 (5): 593611.

Granovetter M. 1985. Economic action and social structure: the problem of embeddedness, American journal of sociology, 91, (3): 481-510.

Haddon L. 2011. Domestication Analysis, Objects of Study, and the Centrality of Technologies in Everyday Life? Canadian Journal of Communication, 36: 311-323.

Hugon P. 1991. L'économie du développement, le temps et l'histoire, Revue économique, 42 (2) : 339-364.

Kamel K, Matthewman R. 2008. The non-transport impacts of High-Speed Trains on regional economic development: a review of the literature. Locate in Kent.

Klein O., Claisse G. 1997. Le TGV-Atlantique : entre récession et concurrence. In Etudes et Recherches, LET, Lyon.

Klein O. 1998. Les modifications de l'offre de transport : Des effets automatiques à l'innovation sociotechnique. L'exemple de la grande vitesse, Espaces et Sociétés, 95 : 95-126.

Mannone V. 1995. L’impact régional du TGV Sud-est. Thèse de Doctorat de Géographie, 2 tomes, Université de Provence Aix-Marseille.

Mérenne-Shoumaker B, Moyart L (2007) Les dynamiques de localisation des services aux entreprises. In Gallouj C, Leloup F, Mérenne-Schoumaker B et Moyart L (eds), Services aux entreprises et développement régional, Bilan et perspectives, De Boeck

Mohino I., Loukaitou-Sideris A. \& Urena J-M. 2014. Impacts of High-Speed Rail on Metropolitan Integration: An Examination of London, Madrid and Paris, International Planning Studies, 306-334.

Nash C. 2009. Quand investir dans des lignes à grande vitesse ?. International transport research symposium-Madrid, OCDE, FIT, Discussion Paper 2009-16.

North D. C. 1991. Institutions, The Journal of Economic Perspectives, 5 (1) (Winter): 97-112.

Pagliara F., La Pietra A., Gómez J., Vassallo J.M., 2015. High-speed rail and the tourism market: evidence from the Madrid case study, Transport Policy, 37: 187-194.

Pagliara F., Delaplace M., Cavuoto R. 2016. Mixed High-Speed, conventional and metro central rail stations as places to work: the case study of Naples, Open Transportation journal, 10: 108-118.

Paulo C. 2007. Une mesure des inégalités de mobilité et d'accès au volant, EspacesTemps.net, Travaux, 27.05.

http://www.espacestemps.net/articles/une-mesure-des-inegalites-de-mobilite-et-acces-au-volant/

Proulx S., Rueff J., Lecomte N. 2007. Une appropriation communautaire des technologies numériques de l'information, UQAM, Mars.

Urena J., Menerault P., Garmendia M. 2009. The high-speed rail challenge for big intermediate cities: a national, regional and local perspective. Cities, 26 (5): 266-279.

Vickerman R-W. 1997. High-speed rail in Europe experience and issues for future development. Annals of Regional Science, 31: 21-38.

De Vries E. J. 2006. Innovation in services in networks of organizations and in the distribution of Services. Research Policy, 35:1037-1051.

Windrum P. and Garcia-Goni M. 2008. A neo-Schumpeterian model of health services innovation, Research Policy, 37: 649-672.

Woessner R. 2014. L'Europe de la grande vitesse ferroviaire, diversités nationales et logiques métropolitaines, Géoconfluences, mis en ligne le 4 novembre 2014. 
Wernerfelt B. 1984. A Resource-Based View of the Firm, Strategic Management Journal, 5, (2) (Apr. - Jun.): 171-180. 